\title{
Corneal Cross-Linking as a Treatment for Fungal Keratitis Associated with Corneal Melting
}

\author{
Vadim Igal $^{a, b}$ Yael Sara Pikkel Igal ${ }^{a, b} \quad$ Yoav Yechezkel Pikkel ${ }^{c}$ \\ ${ }^{a}$ Faculty of Medicine, Bar-Ilan University, Safed, Israel; ${ }^{b}$ Department of Ophthalmology, \\ Ziv Medical Center, Safed, Israel; ' ${ }^{C}$ Carmel Medical Center, Haifa, Israel
}

\section{Keywords}

Corneal cross-linking · Fungal keratitis · Corneal melting

\begin{abstract}
We present the case of a young male that suffered from fungal corneal abscess and corneal melting. The patient was treated successfully by corneal cross-linking. Taking into account our experience from this case, we offer to consider corneal cross-linking as a therapeutic option in cases of fungal keratitis.

\section{Introduction}

Corneal cross-linking (CXL) is a technique that uses the potential of ultraviolet $\mathrm{A}$ light to cross-link tissues in the presence of the nontoxic photosensitizing agent riboflavin. The therapeutic potential of this method was observed by Seiler and colleagues [1] in 1998 who found it to be useful for strengthening the corneal stroma. The concept was based on the observation that naturally occurring protein cross-linking, which accelerates with age, strengthens and stiffens the cornea. Seiler's results laid the cornerstone to the larger-scale study performed by Caporossi et al. [2] who assessed the effectiveness of CXL in reducing the progression of keratoconus and in improving visual acuity in patients with progressive keratoconus. Nowadays, CXL is vastly used to treat keratoconus. 
A few case studies have showed the attempt to use CXL as a treatment for microbial keratitis, with the conclusion that CXL seems to be an effective procedure in the management of superficial microbial keratitis. The attempt to resolve fungal keratitis fell short, with only $50 \%$ of the patients showing improvement and resolution of the keratitis [3].

We report the case of a young patient presenting with fungal keratitis causing corneal melts, who was successfully treated by CXL.

\section{Case Presentation}

A 36-year-old man was admitted to our hospital due to severe pain in his left eye. He mentioned that the pain had begun along with redness 2 days prior to his admission.

On admission, he presented with eyelid swelling, conjunctival erosion, and corneal filtrate at 6 o'clock of a size of $3 / 0$ over $3.2 \mathrm{~mm}$. The corneal abscess location was in the midperiphery making any future corneal graft attempt challenging. There were flare and cells in the anterior chamber and the pupil was hardly seen. Ultrasound examination showed a clear vitreous and no involvement of the posterior segment. The right eye was normal with no pathology.

Anamnesis was taken in depth. It turned out that as part of his work, the patient was peeling plaster off of a wet wall that had fungi on it, and that a small piece of plaster had splashed into his left eye.

He was diagnosed as suffering from fungal keratitis, and further laboratory tests using PCR confirmed the infection to be caused by Fusarium solani. He was then treated with voriconazole, however without significant improvement (Fig. 1).

A couple of days later, a corneal melting process was observed and as the corneal melting continued, it was decided to try treatment with CXL. The patient underwent a CXL treatment and shortly thereafter, the abscess shrank, with significant improvement in symptoms and vision (Fig. 2). The corneal melting stopped after the CXL treatment. The patient was treated for about 3 months with voriconazole.

The following year after the infection, corneal transplantation was done due to severe scarring of the cornea. Today, about 2 years later, best corrected visual acuity in the left eye is $20 / 80$. The corneal graft is clear and shows good indentation with no signs of rejection. The anterior chamber is deep and clear, and other than a mild minimal nuclear cataract. The anterior segment is normal. The optic disc is normal and the C/D ratio is 0.4 . The retina is attached and fundus examination revealed no pathology in the posterior segment.

\section{Discussion}

The use of CXL is very common due to the treatment ability to stabilize the cornea mainly in cases of keratoconus or corneal ectasia. The use of CXL in bacterial keratitis, mainly in cases characterized by corneal melting, has been suggested before and reported as partly successful.

In our case, the use of the CXL technique prevented the continual melting of the cornea caused by fungal keratitis, and eventually allowed corneal transplantation and preservation of the eye's viability and function. Though the cornea in our patient was significantly scarred, this scarring actually enabled corneal grafting since the corneal abscess was large and marginal (towards 6 o'clock). The scar in that region actually preserved recipient tissue and pre- 
vented vascularization and the rejection process origin from the lower limbus. The use of CXL in treating corneal abscesses was described in only a few previous case reports and CXL was described even to a lesser extent in treating fungal corneal abscesses. Though relatively uncommon, fungal keratitis with or without corneal melting does occur and is usually difficult to treat. Taking into account our experience from this case, we offer to consider CXL as a therapeutic option in cases of fungal keratitis. CXL may be an important tool in treating fungal corneal abscess, especially in cases like ours where the corneal abscess is large and peripheral.

\section{Statement of Ethics}

The authors have no ethical conflicts to disclose.

\section{Disclosure Statement}

The authors have nothing to disclose.

\section{References}

1 Spoerl E, Huhle M, Seiler T: Induction of cross-links in corneal tissue. Exp Eye Res 1998;66:97-103.

2 Caporossi A, et al: Parasurgical therapy for keratoconus by riboflavin-ultraviolet type A rays induced cross-linking of corneal collagen: preliminary refractive results in an Italian study. J Cataract Refract Surg 2006;32:837-845.

-3 Shetty R, et al: Collagen crosslinking in the management of advanced non-resolving microbial keratitis. Br J Ophthalmol 2014;98:1033-1035.

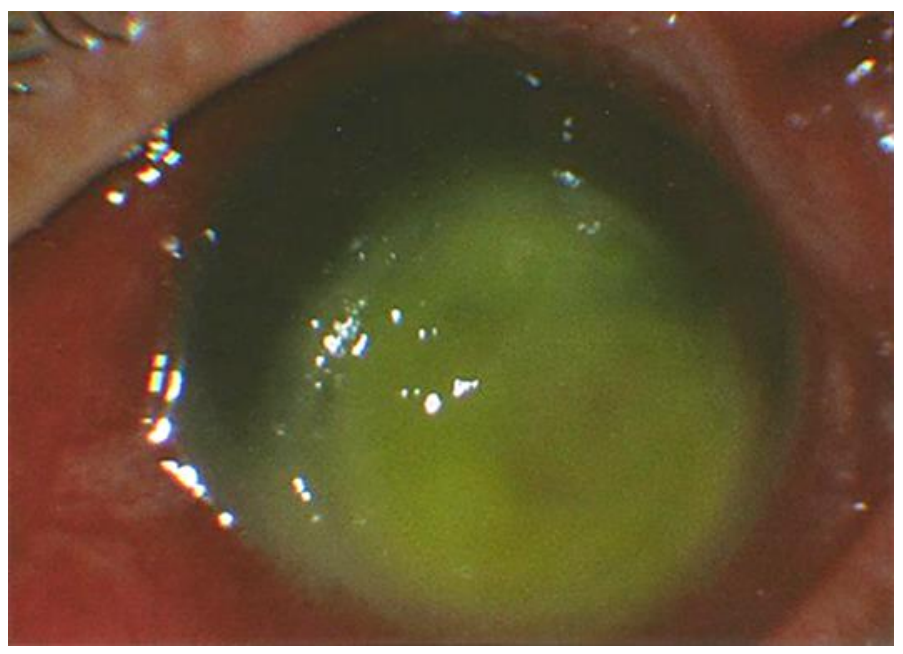

Fig. 1. Prior to the cross-linking treatment. 


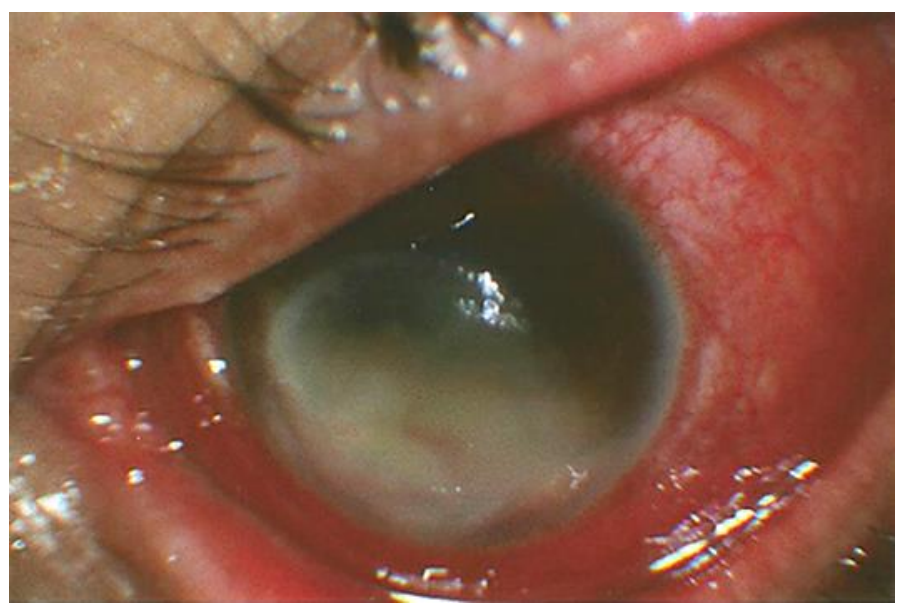

Fig. 2. One week after the cross-linking treatment. 